\title{
A study of perceptual development for musical tuning
}

\author{
MICHAEL P. LYNCH \\ Purdue University, West Lafayette, Indiana \\ and \\ REBECCA E. EILERS \\ University of Miami, Coral Gables, Florida
}

\begin{abstract}
Musical tuning perception in infancy and adulthood was explored in three experiments. In Experiment 1 , Western adults were tested in detection of randomly located mistunings in a melody based on musical interval patterns from native and nonnative musical scales. Subjects performed better in a Western major scale context than in either a Western augmented or a Javanese pelog scale context. Because the major scale is used frequently in Western music and, therefore, is more perceptually familiar than either the augmented scale or the pelog scale are, the adults' pattern of performance is suggestive of musical acculturation. Experiments 2 and 3 were designed to explore the onset of culturally specific perceptual reorganization for music in the age period that has been found to be important in linguistically specific perceptual reorganization for speech. In Experiment 2, 1-year-olds had a pattern of performance similar to that of the adults, but 6month-olds could not detect mistunings reliably better than chance. In Experiment 3, another group of 6-month-olds was tested, and a larger degree of mistuning was used so that floor effects might be avoided. These 6-month-olds performed better in the major and augmented scale contexts than in the pelog context, without a reliable performance difference between the major and augmented contexts. Comparison of the results obtained with 6-month-olds and 1-year-olds suggests that culturally specific perceptual reorganization for musical tuning begins to affect perception between these ages, but the 6-month-olds' pattern of results considered alone is not as clear. The 6-month-olds' better performance on the major and augmented interval patterns than on the pelog interval pattern is potentially attributable to either the 6-month-olds' lesser perceptual acculturation than that of the 1-year-olds or perhaps to an innate predisposition for processing of music based on a single fundamental interval, in this case the semitone.
\end{abstract}

Development of cultural specificity in perception has received considerable attention in speech perception research, but comparatively little is known about the development of culturally specific knowledge for music. A number of studies have demonstrated that better discrimination of native than of nonnative speech contrasts generally occurs by at most 1 year of age (e.g., Kuhl, Williams, Lacerde, Stevens, \& Lindblom, 1992; Werker, Gilbert, Humphrey, \& Tees, 1981; Werker \& Tees, 1984). In studies of early linguistic experience, adult discrimination of native and nonnative speech contrasts has

This research was supported by Grant HD28527 from the National Institute of Child Health \& Human Development (M.P. Lynch, Principal Investigator). The assistance of the infants, their parents, and the adult subjects was greatly appreciated. Preparation of this report was facilitated by helpful commentary from several anonymous reviewers. R.E.E. is affiliated with the Departments of Psychology, Pediatrics, and Otolaryngology at the University of Miami, Mailman Center for Child Development. Correspondence about this article should be addressed to M. P. Lynch, Department of Audiology \& Speech Sciences, 1353 Heavilon Hall, Purdue University, West Lafayette, IN 47907-1353 (bitnet: mlynch@purccvm; internet: mlynch@mace.cc.purdue.edu). been used as a comparison model for the interpretation of infant data. Adult perceivers are generally better discriminators of native than of nonnative speech contrasts, presumably because of linguistic experience. When similar patterns of performance have been found for infants, analogous interpretations have been offered, and dissimilar performance in infants and adults has generally been interpreted as due to infants' lack of perceptual experience.

Lynch, Eilers, Oller, and Urbano (1990) compared adults and infants in a study of musical tuning perception. The term musical tuning refers to the acoustic frequency relations (intervals) among pitch classes. Musical intervals are formalized cross culturally in scales (see Hulse \& Page, 1988; Nettl, 1956). A scale typically consists of 5-7 notes within an octave (Dowling \& Harwood, 1986). Although scales are used throughout the world, there is cross-cultural variety in their tuning (Malm, 1977).

For example, the scales of Western music are based on the semitone (semitone $=$ frequency ratio of $2^{1 / 12}$ or 1.0595). Thus, all Western musical intervals are either semitones or semitone multiples. In contrast, Javanese scales are based on intervals that are not referenced to 
a single frequency ratio or to any mathematical rules. These scales comprise tunings that appear to have been generally agreed upon by the community of musicians in the various geographical regions within the country and are maintained in groups, or gamelan, of similarly tuned instruments (Kunst, 1973). Kunst has documented the tuning of many of the Javanese gamelan.

Because the Western major scale is pervasive in Western music, and because Javanese scales are rarely heard outside of Java, testing Western infants' and adults' abilities to detect mistunings in interval patterns from Western and Javanese scales provides a means of investigating musical acculturation. Following the logic of research on the study of linguistic specificity in infants, if subjects are better detectors of alterations in Western as opposed to Javanese interval patterns, it could be inferred that acculturation has occurred (e.g., Dowling, 1978).

Lynch et al. (1990) found that adult performance differed across native and nonnative musical interval contexts but that infant performance did not. Western adults and 6-month-olds were tested in the detection of mistunings in Western major, Western minor, and Javanese pelog interval patterns. The mistunings were increases in the frequency of the fifth note of a melody. The adults' performance was reliably better than chance in all three interval contexts, and it was reliably better in the Western contexts than in the Javanese context. The infants' performance, although reliably better than chance in all three interval contexts, was not reliably different across contexts, suggesting that musical acculturation had not influenced performance. Therefore, it appeared that the adults had developed, but that the 6-month-olds had not yet developed, sufficient cognitive schemata (Krumhansl, 1990; Shepard \& Jordan, 1984) for musical intervals to affect mistuning perception. Developmentally, the first evidence of musical acculturation as reflected by mistuning perception may, therefore, be evident between 6 months and adulthood.

Although many studies have suggested that acculturation plays a role in musical processing (see Castellano, Bharucha, \& Krumhansl, 1984; Dowling \& Harwood, 1986; Kessler, Hansen, \& Shepard, 1984; Krumhansl, 1990), the onset of cultural specificity for music perception has been sparsely documented (see, e.g., Cohen, Thorpe, \& Trehub, 1987; Krumhansl \& Keil, 1982; Trehub, Cohen, Thorpe, \& Morrongiello, 1986). In particular, Lynch et al.'s (1990) study did not address an important stimulus issue; the use of the interval patterns from major, minor, and pelog scales did not necessarily allow for clear acculturation interpretations. Because the intervallic structures of both the major and the minor scales are based on patterns of intervals (semitones and whole tones; whole tone $=2$ semitones) that are referenced to the semitone (major: whole tone, whole tone, semitone, whole tone, whole tone, whole tone, semitone; minor: whole tone, semitone, whole tone, whole tone, whole tone, semitone, whole tone), their interval structures are less complex than that of the pelog scale. Therefore, better adult performance on both the major and the minor scales than on the pelog scale might have been predicted independently of listening experience because of the simpler interval structures of the Western scale contexts (see Balzano, 1980, 1982, 1986a, 1986b, 1987, for detailed treatment of potential advantages of musical systems based on a fundamental interval).

Lynch, Eilers, Oller, Urbano, and Wilson (1991) attempted to address this issue by replacing the minor scale with the Western augmented scale in testing adult subjects. Although the dependent variable in this study was the just noticeable difference (JND) for mistuning, rather than the accuracy of mistuning detection, the overall pattern of findings is relevant here. The augmented scale is similar to the major scale because it is based on the semitone and semitone multiples. The augmented scale, however, is a symmetrical, and therefore predictable, arrangement of intervals, because it is made up of alternating semitones and minor thirds ( 3 semitones), potentially facilitating its processing in comparison with processing of the major scale. If the complexity of interval patterns is a dominant influence on processing, performance on an augmented interval pattern should have ranked highest because it is based on the semitone and comprises a predictable sequence of intervals. Performance on a major interval pattern should have been second best because, although it is based on the semitone, its pattern of intervals is not as predictable as that of the augmented scale. Performance on a pelog interval pattern should have been worst because it is not based on the semitone and it does not comprise a predictable interval sequence. This predicted order was not obtained, however. Performance was best on a major interval pattern, as reflected by a reliably lower JND for mistuning in this pattern than in either an augmented or a pelog interval pattern. The subjects' JNDs for the augmented and pelog interval patterns were statistically equivalent. These results suggest an acculturation effect because of the substantially more prevalent use of the major scale than of the augmented scale in Western music.

The present study was designed to improve on the stimuli, experimental design, and analyses of the Lynch et al. (1990) study, partly through use of the augmented scale as in Lynch et al. (1991). The stimuli were further improved by randomly changing the location of the mistuning within the melody across trials. In the Lynch et al. (1990) study, the mistunings always occurred on the fifth note of the stimulus melody. Therefore, subjects could have ignored the surrounding musical context in their detection of mistunings. In the present study, the mistuned note was randomly selected across trials, making its serial location unpredictable and requiring the subjects to attend to the entire melody.

The other design improvements were made in subject selection and adult testing. Subject selection was improved by including 1-year-olds. This age group, in addition to 
6-month-olds and adults, was selected on the basis of the possibility that the time course of perceptual learning for music might parallel that for speech: a number of findings have suggested that perceptual reorganization for speech occurs by the end of the lst year of life (e.g., Werker et al., 1981). Adult testing was improved by adding nonauditory distraction to the music perception task that would control for ceiling effects. This measure was taken because testing of infants and adults on equivalent stimuli is desirable but often results in a task that is very easy for adults.

\section{EXPERIMENT 1}

\section{Method}

Subjects. Thirty Western adults participated, and they were randomly assigned to three groups of 10 subjects each. All subjects were at least 19 years old $(M=30.3$ years; $S D=6.5$ years; 15 females, 15 males) and had little or no formal training in music. The subjects reported that they were experiencing no difficulties with their hearing and that to their recollection they had no listening experience of non-Western music.

Stimuli. The stimulus melody was based on interval patterns from the Western major, Western augmented, and Javanese pelog musical scales. The major scale is the basis for much of Western music, so it is often heard in Western culture. The augmented scale is also from Western music, but it is seldom heard. It is primarily used by jazz musicians in improvisation (e.g., the music of Herbie Hancock and Wayne Shorter). The pelog scale is rarely heard outside of Java. The frequencies of the melody notes for each scale context are shown in Table 1.

The intervals of both the major and augmented scales are derived from the semitone ( $S T=1.0595$ frequency ratio). The major scale comprises STs and whole tones (WT $=2$ semitones) in an asym- metrical arrangement: WT, WT, ST, WT, WT, WT, ST. In contrast with the major scale, the augmented scale comprises a symmetrical arrangement of alternating minor thirds ( $\mathrm{m} 3=3$ semitones) and semitones: m3, ST, m3, ST, m3, ST (see Campbell, 1988, for a comprehensive description of the augmented scale and its uses). Therefore, although they are both based on the ST and ST multiples, the interval patterns of the major and augmented scales are different.

The intervals of the pelog scale (Kunst, 1973, p. 573) are different from those of both the major and the augmented scales; most importantly, perhaps, they are not derived from a single frequency ratio. The first 5 notes of each scale were used in the test melody (Notes $1,2,3,4,5,3,1$ ), creating a melodic pattern with consistent frequency ranges, the same up-down melodic contour, and the same timing characteristics across the major, augmented, and pelog interval contexts. Thus, the only systematic differences among the major, augmented, and pelog testing conditions were the frequency interval patterns.

In the stimulus melody, individual tones were $300 \mathrm{msec}$ long, with $20 \mathrm{msec}$ each of linear attack and decay. The onsets of Notes 1-5 occurred at 500-msec intervals, and the onsets of Notes 5-7 occurred at $1-\mathrm{sec}$ intervals, providing the melody with a rhythmic quality within an overall duration of $4.3 \mathrm{sec}$. Each note was a summation of two sine waves, the fundamental frequency $(F 0)$ and the second harmonic $(\mathrm{H} 2)$ an octave above (frequency ratio of $2: 1$ ). An IBM PS/2 microcomputer generated digital representations of $F 0$ and $\mathrm{H} 2$ (amplitude ratio of $7: 3$ ) that were summed and then processed by digital-analog conversion. The amplifier was set to output each tone at $75 \mathrm{~dB}$ SPL in the soundfield, and the ambient noise level in the testing chamber was approximately $15 \mathrm{~dB}$ (AScale, Bruel \& Kjaer sound level meter, Type 2203).

Apparatus. The experimental sessions were conducted in a double-walled, sound-attenuated booth containing a high-fidelity loudspeaker (Pioneer HPM 100) and a computer-monitored response panel. The experimenter used this panel to initiate trials and to record the subjects' responses. Additional control mechanisms, including

Table 1

Frequency Values of Melody Notes (Ratios and Intervals relate to the First Note)

\begin{tabular}{|c|c|c|c|c|}
\hline Note & $\begin{array}{c}\text { Frequency } \\
\text { (cps) }\end{array}$ & $\begin{array}{c}\text { Frequency } \\
\text { Ratio }\end{array}$ & $\begin{array}{c}\text { Interval } \\
\text { (cents) }\end{array}$ & $\begin{array}{l}\text { Note } \\
\text { Name }\end{array}$ \\
\hline \multicolumn{5}{|c|}{ Major } \\
\hline 1 & 293.7 & 1.0000 & 0 & D4 \\
\hline 2 & 329.6 & 1.1225 & 200 & E4 \\
\hline 3 & 370.0 & 1.2598 & 400 & $F \# 4$ \\
\hline 4 & 392.0 & 1.3349 & 500 & G4 \\
\hline 5 & 440.0 & 1.4981 & 700 & A4 \\
\hline 6 & 370.0 & 1.2598 & 400 & $F \# 4$ \\
\hline 7 & 293.7 & 1.0000 & 0 & D4 \\
\hline \multicolumn{5}{|c|}{ Augmented } \\
\hline 1 & 293.7 & 1.0000 & 0 & D4 \\
\hline 2 & 349.2 & 1.1890 & 300 & F4 \\
\hline 3 & 370.0 & 1.2598 & 400 & $F \# 4$ \\
\hline 4 & 440.0 & 1.4981 & 700 & A4 \\
\hline 5 & 466.2 & 1.5873 & 800 & A\#4 \\
\hline 6 & 370.0 & 1.2598 & 400 & $F \# 4$ \\
\hline 7 & 293.7 & 1.0000 & 0 & D4 \\
\hline \multicolumn{5}{|c|}{ Pelog } \\
\hline 1 & 293.7 & 1.0000 & 0 & bem \\
\hline 2 & 311.7 & 1.0613 & 103 & gulu \\
\hline 3 & 341.2 & 1.1617 & 259 & dada \\
\hline 4 & 390.7 & 1.3303 & 494 & pelog \\
\hline 5 & 436.2 & 1.4852 & 685 & lima \\
\hline 6 & 341.2 & 1.1617 & 259 & dada \\
\hline 7 & 293.7 & 1.0000 & 0 & bem \\
\hline
\end{tabular}


an IBM AT personal computer and an amplification system (Accuphase E202), were in an anteroom. Feedback was provided to the subjects by four animated toys in four separate compartments concealed in a smoked Plexiglas box. The computer randomly activated one of the four toys and illuminated one compartment for $2.5 \mathrm{sec}$ or until the end of the stimulus, whichever was later. Videotaped silent cartoons were shown on a Sony color television (14-in. screen).

Procedure. An adaptation for adults of the operant head-turn procedure (OHTP; Eilers \& Gavin, 1981; Eilers, Wilson, \& Moore, 1977 ) was used to test perception of mistunings in the major, augmented, and pelog interval patterns. This procedure was the same as that used with adults in Lynch et al. (1990), and it was designed to be as similar as possible to that used in testing of infants in Experiments 2 and 3 . The differences between the adult and infant procedures were that adults indicated responses with a hand-raise (infant responses are head-turns), and some of the adults were tested in conditions of nonauditory distraction, which were incorporated as controls for ceiling effects. Testing consisted of three sessions, in each of which one of the interval patterns was the basis of the melody.

In test sessions, the well-tuned version $(\mathrm{Sb})$ of one of the interval patterns was played repeatedly through a loudspeaker. Sb was presented continuously except when the frequency of one of its notes was changed, resulting in presentation of a mistuned version (Sd) of the melody. Sessions began with a shaping phase in which Sd was accompanied by 12-, 8-, and 4-dB intensity changes from $\mathrm{Sb}$. Two trials were presented at each intensity level, and subjects responded correctly to these trials with $99 \%$ accuracy overall. This level of performance was consistent across the three testing contexts.

Shaping was followed by 30 equal-intensity trials. One half were change trials in which the frequency of a randomly selected melody note (excluding the first note) was increased by $2.8 \%$, and the remaining were no-change trials. No-change trials are crucial in administration of the OHTP, because data obtained in them allow for assessment of the degree to which responding was under stimulus control. Change and no-change trials were randomly intermingled within each session. The experimenter initiated trials by pressing a button on a concealed panel. If a subject responded during a trial interval ( 2 repetitions of either $\mathrm{Sb}$ during no-change trials or of $\mathrm{Sd}$ during change trials) by raising a hand, the experimenter pressed a button to record the response. Only responses during change trials were followed by visual feedback in the form of activation of one of the mechanical toys. Hand-raises during no-change trials were recorded in order to document responding that was not under stimulus control.

All three of the test sessions conducted with each adult were completed in one visit of about an hour. The three adult groups differed in the level of nonauditory distraction that they received during sessions. This distraction was incorporated into the study because the same stimuli were to be used with infant subjects in Experiment 2 . Therefore, the $2.8 \%$ mistuning was expected to be very easy for adults to detect (e.g., see Lynch et al., 1990), and distraction was used in an attempt to prevent ceiling effects. A no-cartoons group received no distraction during sessions. A cartoons group watched silent, action-based cartoons (e.g., "Bugs Bunny" and “'Road Runner') during sessions, and they were told to pay close attention to the cartoons because a quiz on the content of the cartoons would be given following the session (a quiz was not actually given). A cartoons + $Q$ group both watched the same silent cartoons as the cartoons group did and referred to a list of questions about the cartoons, on which they were quizzed following the session. The cartoons $+Q$ group's quiz was conducted at the conclusion of their 1-h visit.

The instructions received by all of the adult groups prior to each session were to "make the toy come on by raising a hand when you hear the melody change." The subjects were not given any additional hints or verbal assistance with the task, but they reported that the shaping phase at the beginning of each session made the task demands clear.

Precautions were taken to control experimenter bias and potential effects of stimulus order. During training and testing sessions, the experimenter wore earplugs, as well as headphones through which masking music was played. Pilot trials demonstrated that the experimenter thus masked was unable to detect mistunings above chance; therefore, he was incapable of systematically biasing subjects' headturning or handraising responses. Additionally, the order of testing on the major, augmented, and pelog interval patterns was counterbalanced across subjects within each group.

\section{Results}

Dependent variable. As introduced elsewhere (Thorpe \& Trehub, 1989; Thorpe, Trehub, Morrongiello, \& Bull, 1988; Trainor \& Trehub, 1992), the OHTP lends itself to signal detection analysis, because the infants' task is to detect a "signal" (mistuned version of melodies) embedded within a surrounding auditory context (repeated well-tuned version of melodies). As noted by Thorpe et al. (1988), this go/no-go procedure corresponds to the adult vigilance (yes/no) task (see Green \& Swets, 1966). Yes/no tables from signal detection theory (Swets, 1964, pp. 659678) can, therefore, be used to convert hit rates (proportion of change trials during which subjects performed a hand-raise) and false alarm rates (proportion of no-change trials during which subjects performed a hand-raise) into $d^{\prime}$ scores. This conversion provides the dual advantages of minimizing response bias and normalizing score distributions (discussed in Green \& Swets, 1966; Massaro, 1975, pp. 119-141). Because the use of $d^{\prime}$ as an index of performance in the OHTP is a relatively new development, the percentage correct scores and corresponding standard deviations are also reported in the data figures so that performance in the present task might be compared with that in other studies done with the same paradigm.

Because subjects occasionally do not commit errors on change trials (reflected by a hit rate of 1.0 ) and on nochange trials (reflected by a false alarm rate of 0.0 ), resulting in a statistically infinite $d^{\prime}$, all proportions were transformed before determination of $d^{\prime}$ scores. Because of the small number of trials conducted in each session, infinite $d^{\prime}$ scores in the present study were presumed to be attributable to sampling error, rather than to the occurrence of infinite $d^{\prime}$ values (see Macmillan \& Kaplan, $1985)$. In order to correct for statistically infinite $d^{\prime}$ scores, hit and false alarm rates were calculated by adding 0.5 to the number of hand-raises (e.g., changing number of hand-raises during change trials from 15.0 to 15.5) and dividing the result by the actual number of change trials plus 1 (e.g., changing the denominator from 15 to 16). This procedure does not change the rank order of scores, and it allows one to avoid inappropriately infinite $d^{\prime}$ values (Trainor \& Trehub, 1992).

Subject performance. Analyses of variance (ANOVAs) were conducted to compare performance across the musical interval patterns and across subject groups, and $t$ tests (one-tailed) were used to evaluate performance in comparison with chance $\left(d^{\prime}=0\right)$. One-tailed $t$ tests were used because only performance that was reliably greater than 
zero was expected. This chance level was used because it is the score that would result from an equal number of hits and false alarms, which is the standard chance level used in studies done with the OHTP (see also Thorpe \& Trehub, 1989; Thorpe et al., 1988; Trainor \& Trehub, 1992).

A 3 (interval pattern) $\times 3$ (group) repeated measures ANOVA was conducted. Statistically reliable main effects of interval pattern $[F(2,54)=22.1, p<.0001\}$ and group $[F(2,27)=11.8, p<.0001]$ were found. The interval pattern $\times$ group interaction was not statistically reliable $[F(4,54)=1.8, p<.148]$.

Figure 1 shows the interval pattern main effect. The subjects performed reliably better than chance on the major $[t(29)=11.2, p<.0005]$, augmented $[t(29)=$ $7.6, p<.0005]$, and pelog $[t(29)=5.3, p<.0005]$ interval patterns. Post hoc analysis indicated that performance on the major interval pattern was reliably better than performance on the augmented and pelog interval patterns (Duncan's range statistic, $p<.01$ ). Performance on the augmented interval pattern was not reliably different from performance on the pelog interval pattern.

Figure 2 shows the group main effect. Performance was reliably better than chance for the no-cartoons $[t(9)=7.1$, $p<.0005]$, cartoons $[t(9)=4.7, p<.005]$, and cartoons $+Q[r(9)=5.6, p<.0005]$ groups. Post hoc analysis revealed that the no-cartoons group performed reliably better than the cartoons and cartoons $+Q$ groups (Duncan's range statistic, $p<.01$ ). The performance of the cartoons group was not reliably different from that of the cartoons $+Q$ group. Performance within the

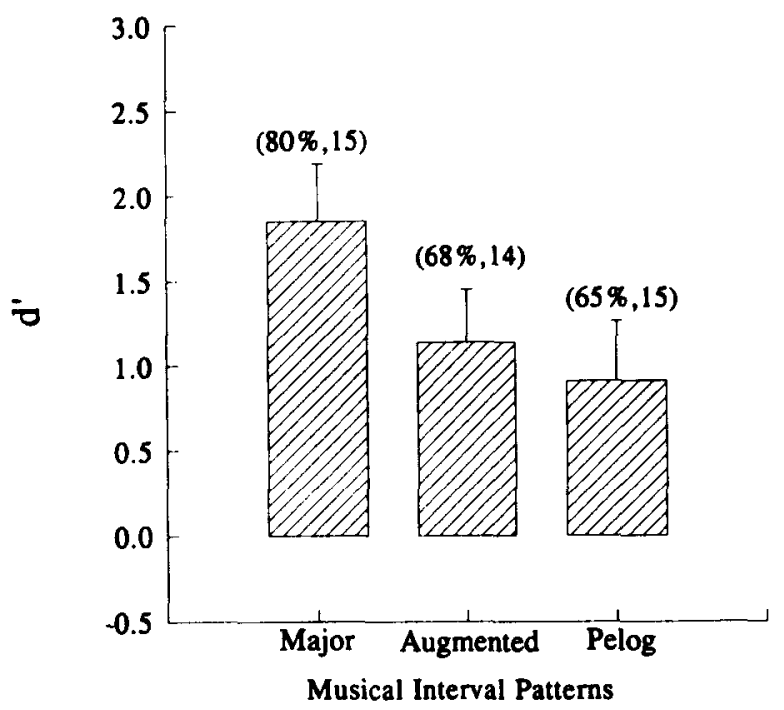

Figure 1. Adult performance in Experiment 1 collapsed across groups on the major, augmented, and pelog interval patterns. The error bars indicate the $95 \%$ confidence interval of each mean. Chance performance in the operant head-turn paradigm is $d^{\prime \prime}=0$. Indicated in parentheses are the percentage correct scores and corresponding standard deviations. Chance performance in percentage correct is $50 \%$.

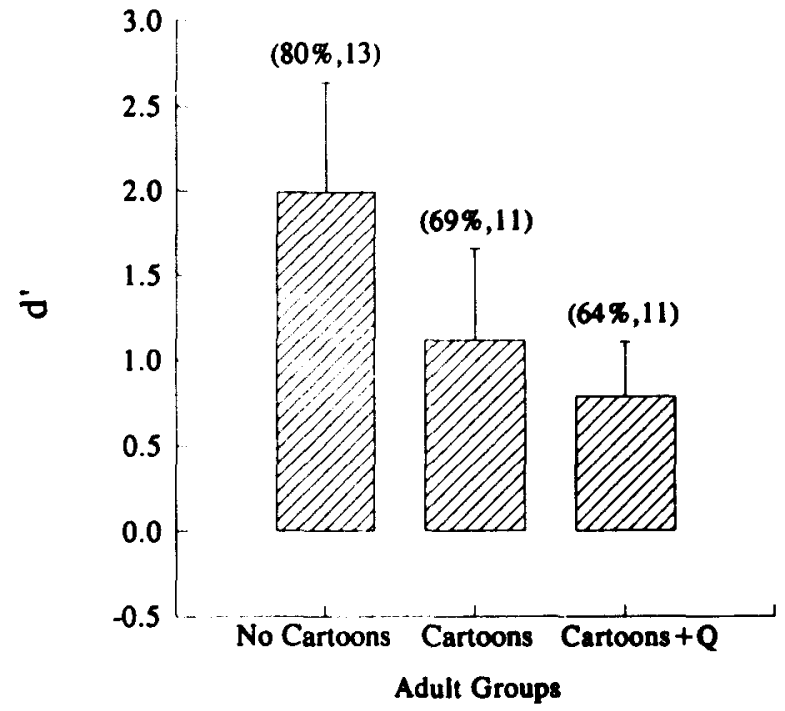

Figure 2. Performance of the three adult groups in Experiment 1, collapeed across musical interval patterns. The error bars ladicate the $95 \%$ confidence interval of each mean. Chance performance in the operant head-turn paradigm is $d^{\prime}=0$. Indicated in parentheses are the percentage correct scores and corresponding standard deviations. Chance performance in percentage correct is $50 \%$.

cartoons $+Q$ group was not correlated with performance on the quiz about the content of the cartoons.

\section{Discussion}

The better detection of mistunings in the major context than in the augmented and pelog contexts suggests that musical acculturation influenced performance. In addition, processing uncertainty introduced by the nonauditory distraction conditions was useful for the avoidance of ceiling effects. Cohen, Trehub, and Thorpe (1989) found similar effects of processing uncertainty when they altered the structural complexity of melodic sequences.

The acculturation effect in the present experiment replicates Lynch et al. (1991), but with the use of a different dependent variable (discrimination accuracy instead of just noticeable difference) (see also Cohen et al., 1989). The intervals of the major and augmented contexts are based on the Western semitone and its multiples. In addition, the interval pattern of the augmented scale is predictable because it consists of alternating minor 3 rds and semitones. Although the simpler interval pattern of the augmented scale might have led to better performance on it than on the major scale, the opposite result was found, suggesting learned cultural specificity of perception. Experiment 2 was designed to examine whether the onset of this cultural specificity, like that of speech, occurs in infancy.

\section{EXPERIMENT 2}

\section{Method}

Subjects. Twenty healthy Western infants participated in this experiment. All parents reported that their children had been exposed 
Table 2

Training Melody for Infants

\begin{tabular}{cccc}
\hline Note & $\begin{array}{c}\text { Frequency } \\
\text { (cps) }\end{array}$ & $\begin{array}{c}\text { Frequency } \\
\text { Ratio }\end{array}$ & $\begin{array}{c}\text { Interval } \\
\text { (cents) }\end{array}$ \\
\hline 1 & 293.7 & 1.0000 & 0 \\
2 & 314.5 & 1.0710 & 119 \\
3 & 350.0 & 1.1920 & 304 \\
4 & 400.0 & 1.3620 & 535 \\
5 & 412.0 & 1.4060 & 590 \\
6 & 350.0 & 1.1920 & 304 \\
7 & 293.7 & 1.0000 & 0 \\
\hline
\end{tabular}

only to Western music. Ten of the infants were 10-13 months old at the beginning of the study ( $M=11$ months, 20 days; $S D=46$ days; 4 females, 6 males), and 10 of them were 5-7 months old ( $M=6$ months, 25 days; $S D=24$ days; 5 females, 5 males). Because experimental sessions were conducted on a weekly basis, these groups were an average of 12 months, 27 days ( $S D=49$ days) and 7 months, 27 days ( $S D=34$ days) old, respectively, by the end of their participation in the study. Sixteen 5-7-month-olds were originally recruited to participate. Three of these infants did not pass training, and 3 of them did not finish the testing phase because they did not appear for their scheduled testing appointments. Eleven 10-13-month-olds were originally recruited. Although they all passed training, 1 subject did not complete the testing phase because of nonreport for scheduled appointments.

Stimuli. Before testing on the three interval patterns from Experiment 1 , infants were trained in the OHTP with a quasirandomly determined interval pattern that was unrelated to the major, augmented, and pelog interval patterns used in testing. The acoustic frequencies and frequency relations for this interval pattern are shown in Table 2. As in Experiment 1, mistunings occurred across trials in randomly selected melody notes (except the first note).

Apparatus. The apparatus from Experiment 1 was also used in this experiment.

Training procedure. The OHTP was successively used in the training and testing phases. The training phase consisted of a maximum of three sessions in which infants learned to detect $5.0 \%$ mistunings in the random interval pattern before testing on the major, augmented, and pelog patterns. This amount of mistuning was larger than that used in the testing sessions, because previous studies (e.g., Lynch et al., 1990) suggested that it would easily be detectable by the infants.

In training sessions, the well-tuned random pattern $(\mathrm{Sb})$ was played repeatedly through a loudspeaker. Sb was presented continuously, except when the frequency of one of its notes was changed (Sd). Training sessions began with a shaping phase, in which Sd was accompanied by 12-, 8-, and 4-dB intensity changes from Sb. During presentation of $\mathrm{Sb}$, the experimenter, who was sitting across a table from the infant, engaged the infant with quiet, hand-held toys. The infants sat in a parent's lap during sessions. If a subject did not perform a head-turn toward the loudspeaker in response to the 12-dB change after two consecutive presentations, the visual reinforcer, which was located on top of the speaker, was activated toward the end of Sd. Infants required at the most two initiations of responses with activation of an electronic toy to establish a connection between the change to Sd and the appropriate response. Four trials were presented at each intensity level. Shaping was followed by equal-intensity trials. One half of these trials were change trials in which the frequency of a randomly selected melody note was increased by $5.0 \%$, and the remaining trials were no-change trials.

The experimenter initiated the trials by pressing a button on a concealed panel. If an infant responded during a trial interval, the experimenter pressed a button to record the response. Only responses during change trials were followed by activation of a visual reinforcer. Correct responses (head-turn during a change trial, no head- turn during a no-change trial) to $9 / 10$ contiguous trials were required for the infants to pass training and proceed to the testing phase of the study.

Testing procedure. The test sessions were, in general, conducted identically to those in Experiment 1 . As in Experiment 1, shaping was followed by 30 equal-intensity test trials, half of which were change trials and half of which were no-change (control) trials. In contrast with the procedure used with the adults, the infants were engaged at midline with toys during presentation of $\mathrm{Sb}$. The infants responded correctly to shaping trials at the beginning of each test session, with $98 \%$ accuracy overall. This level of performance was consistent across the three testing contexts. Sessions were conducted in weekly visits of approximately $20 \mathrm{~min}$, and only 1 training or testing session was conducted during each visit. The controls for experimenter bias and stimulus order effects used in Experiment 1 were also used in this experiment.

\section{Results}

As in Experiment 1, hit and false alarm rates were converted to $d^{\prime}$ scores. These scores were then compared with chance, using $t$ tests (one-tailed) in the same manner as in Experiment 1 and entered into ANOVAs. For compar-

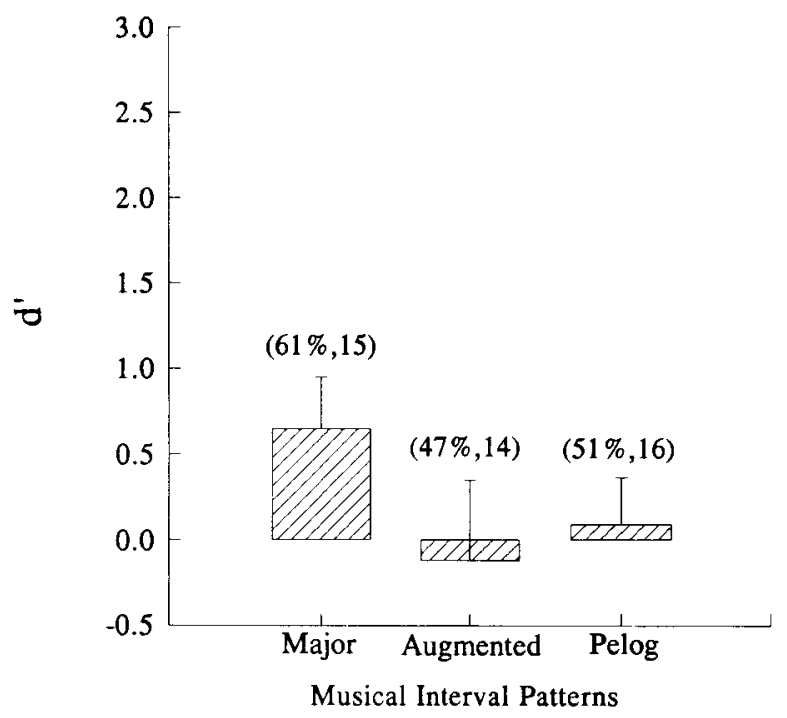

Figure 3. Performance of the 1-year-olds in detection of the $2.8 \%$ mistuning in the major, augmented, and pelog interval patterns in Experiment 2. The error bars indicate the $95 \%$ confidence interval of each mean. Chance performance in the operant head-turn paradigm is $d^{\prime}=0$. Indicated in parentheses are the percentage correct scores and corresponding standard deviations. Chance performance in percentage correct is $50 \%$. 
ison with some previous studies, percentage correct scores and the corresponding standard deviations are indicated on the data figure along with $d^{\prime}$ scores.

Because the 6-month-olds did not perform reliably better than chance on any of the interval patterns ( $d^{\prime}$ scores were, for major $=0.3$, for augmented $=0.11$, and for pelog $=$ 0.09 ; all $t$ tests resulted in $p>.05$ ), Figure 3 shows only the $d^{\prime}$ scores of the 1-year-olds. The 1-year-olds performed reliably better than chance on the major interval pattern $[t(9)=4.85, p<.0005]$, but their performance was not reliably better than chance on the augmented $[t(9)=-0.57, p>.25]$ and pelog $[t(9)=0.72, p>$ .2] interval patterns. Overall, it is apparent that $d^{\prime}$ scores for infants are lower than those commonly found with adult subjects. The present $d^{\prime}$ scores are consistent with those obtained for infants in previous studies (Thorpe \& Trehub, 1989; Thorpe et al., 1988; Trainor \& Trehub, 1992).

A one-way ANOVA indicated that the 1-year-olds' performance was reliably different across the major, augmented, and pelog interval patterns $[F(2,18)=5.4, p<$ $.014]$. Post hoc analysis revealed that, similarly to the adults' performance, the infants' performance on the major interval pattern was reliably better than that on the augmented and pelog interval patterns (Duncan's range statistic was $p<.05$ for the major-augmented comparison and $p<.01$ for the major-pelog comparison).

\section{Discussion}

The performance of the 1-year-olds was similar to that of the adults: better in the major context than in the augmented and pelog contexts. The 6-month-olds, however, were unable to do the task, possibly because the amount of mistuning was too small for them to detect in the musical context, although previous studies have shown it to be detectable by infants of this age in basic frequency discrimination tasks (e.g., Olsho, Schoon, Sakai, Turpin, \& Sperduto, 1982). In Experiment 3, a larger degree of mistuning was used with a different group of 6-month-olds.

\section{EXPERIMENT 3}

\section{Method}

Subjects. Thirteen healthy Western infants participated in this experiment. As in Experiment 2, all parents reported that their children had only been exposed to Western music. The infants were 5-7 months of age at the beginning of the study ( $M=6$ months, 15 days; $S D=20$ days; 7 females, 6 males). In the final testing session, these infants were on the average 7 months, 18 days $(S D=$ 23 days) of age. Fifteen infants were originally recruited, but 2 of them did not pass training.

Stimuli. The training stimuli used in Experiment 2 were also used in this experiment. The test stimuli were also the same, with the exception that melody notes were mistuned by $4.5 \%$, rather than $2.8 \%$.

Apparatus. The equipment from Experiments 1 and 2 was used in this experiment.

Procedure. The procedures from Experiment 2 were also used in this experiment. As in Experiment 2, the infants responded correctly to shaping trials, with $98 \%$ accuracy overall. This level of performance was consistent across the three testing contexts.

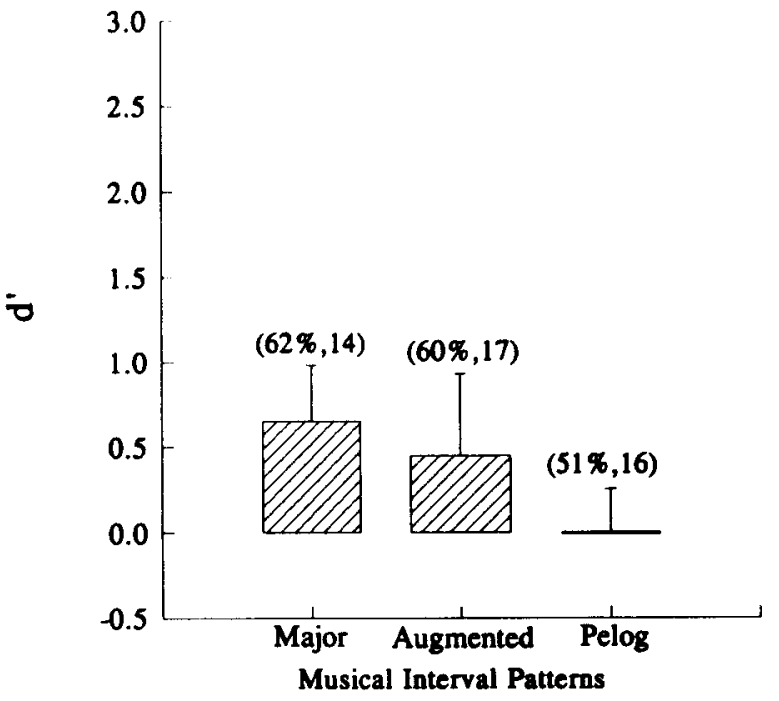

Figure 4. Performance of the 6-month-olds in detection of the $4.5 \%$ mistuning in the major, augmented, and pelog interval patterns in Experiment 3. The error bars indicate the $95 \%$ conflence interval of each mean. Chance performance in the operant bead-turn paradigm is $d^{\prime}=0$. Indicated in parentheses are the percentage correct scores and corresponding standard deviations. Chance performance in percentage correct is $50 \%$.

\section{Results}

As in Experiments 1 and 2, hit and false alarm rates were converted to $d^{\prime}$ scores, and the corresponding percentage correct scores (with $S D$ s) are indicated on the data figure, as well. Performance was compared with chance, using $t$ tests (one-tailed) and entered into ANOVAs.

Figure 4 shows the subjects' $d^{\prime}$ scores. Performance was reliably better than chance on the major $[r(12)=4.3$, $p<.005]$ and augmented $[t(12)=2.0, p<.05]$ interval patterns, but it was not reliably better than chance on the pelog interval pattern $[t(12)=0.07, p>.2]$. A oneway ANOVA indicated that performance was reliably different overall across the major, augmented, and pelog interval patterns $[F(2,24)=4.9, p<.016]$. Post hoc analysis revealed that performance on both the major and the augmented interval patterns was reliably better than that on the pelog interval pattern (Duncan's range statistic $p<$ .05 for the augmented-pelog comparison, $p<.01$ for the major-pelog comparison). Performance on the major interval pattern, however, was not reliably different from performance on the augmented interval pattern.

\section{Discussion}

In contrast with the adults and 1-year-olds, the 6-montholds in this experiment performed equivalently on major and augmented interval patterns, with performance on both of these patterns reliably better than that on the pelog interval pattern. This pattern of results could have at least two viable interpretations. One interpretation could be that because the augmented scale is present in a small proportion of the native music of the infant subjects, per- 
haps its processing was facilitated in comparison with that of the pelog scale as a result of listening experience. The other interpretation could be that because the interval patterns in the major and augmented interval patterns are simpler than those in the pelog interval pattern because of their basis on the semitone, their processing is inherently facilitated in comparison with that of the pelog scale.

There is less ambiguity in the comparison of the results of this experiment with those of Experiment 2. This comparison suggests that between 6 months and 1 year of age, the processing of interval patterns becomes more culturally specific. This finding suggests perceptual reorganization for musical tuning in a developmental period that parallels linguistically specific perceptual reorganization for processing of consonantal speech contrasts.

\section{GENERAL DISCUSSION}

Overall, this study suggests that perception of musical tuning develops toward cultural specificity in the 1st year of life. The 1-year-old infants in this study had a pattern of performance similar to that of the adults: better mistuning detection in the major context than in either the augmented or the pelog context. Six-month-old infants, however, detected mistunings better in the major and the augmented contexts than in the pelog context, and their performance was statistically equivalent in the major and augmented contexts. The more culturally specific effect of the 1-year-olds than of the 6-month-olds suggests that the development of culturally specific perceptual reorganization for music occurs in a developmental period similar to that for the perceptual reorganization of native speech contrasts.

The 1-year-olds' better performance in the major context than in the augmented context agrees with previous work (Cohen et al., 1987), in which 7-11-month-olds were better in detection of semitone changes in transposed major triads than in transposed augmented triads (triads include only notes 1,3 , and 5 of the corresponding scales and are centrally important structures in Western musical harmony). The difference between the present study and that of Cohen et al. is that discrete groups of infants were tested in the present work. Cohen et al. evaluated the 7-11-month age range as a whole. Because separate 6-month-old and 1-year-old groups were tested in the present work, the current study may have allowed for greater resolution of the ontogenetic process of tuning perception and suggests that significant formation of mental representation of musical interval patterns takes place between 6 and 12 months of age.

The comparison between performance in the major and augmented contexts is particularly important because the structure of both scales was well-represented in the stimulus melody in the present study. Therefore, performance differences between the major and augmented contexts can be wholly attributed to the interval patterns, without potential confounding influences of functional significance of the particular notes chosen for the stimulus melody. The selection of notes for the pelog interval pattern (Notes
$1,2,3,4,5,3,1)$ was influenced by the selection of functionally important notes of the major and augmented scales for the stimulus melodies. Because the subjects tested were Western and were screened so that their perceptual experience had occurred only with Western music, representation of the harmony of the Western scale contexts was given priority in stimulus design over representation of pelog note function. Therefore, in order to maintain consistency in melodic contour and frequency ranges across all three melodic contexts, the pelog interval pattern was chosen without regard for its well-formedness within Javanese music. Future studies will include evaluations of the culture-fairness of nonnative musical stimuli; steps will be taken to ensure that these stimuli are "good" (Trehub, Thorpe, \& Trainor, 1990), as defined by their particular musical culture. Two studies have suggested that well-formedness within Western music affects fully acculturated (adult) listeners' processing of melodies (Cuddy, Cohen, \& Mewhort, 1981; Cuddy, Cohen, \& Miller, 1979), providing some empirical support for the importance of this issue in consideration of fully acculturated listeners. Its importance in the consideration of developing listeners has not been explored.

The present findings with 6-month-olds contrast with those of Lynch et al. (1990). The performance of the 6month-olds in the Lynch et al. study was reliably better than chance for Western and Javanese interval patterns, but it was not different across the interval patterns. Trehub et al. (1986) obtained a similar result in their study of 10-month-old infants' perception of major and augmented triads. The Lynch et al. study involved a relatively simple task because the mistuning was always in the same note, but the procedure in the Trehub et al. study was similar to that of the present work, because mistunings in that study were randomly located throughout melodic sequences across trials. The findings of Trehub et al. and of Lynch et al. suggested that 6- and 10-month-olds have not acquired sufficient perceptual knowledge for culturally specific musical interval patterns to influence perception.

In the present study, reliable performance differences across musical interval patterns were found both for 1year-olds and for 6-month-olds. The results for the 6month-olds in the present work may have differed from those of the Lynch et al. (1990) study because of the increased task demand of the randomly located mistuning. The present results may have differed from those of Trehub et al. (1986) for at least two reasons. First, in addition to major and augmented contexts as in the Trehub et al. study, the present study involved a pelog (nonnative) context. Therefore, because the pelog context is fundamentally different from the major and augmented contexts in lack of basis on the semitone or any other fundamental interval, the present study may have tapped information about infants' processing of interval structure not explored by Trehub et al. A second important difference between the present work and that of Trehub et al. is that the present group of 1-year-olds was, on the average, 50 days older than the 9-11-month-olds of the Trehub et al. study were. As indicated in several areas 
of infant development (see, e.g., Johnson \& Morton, 1991), significant changes in infant abilities can occur over such a time span. The present work may have provided a better window on musical acculturation in infancy than the Trehub et al. study did because of its focus on older and younger babies.

In addition to the Lynch et al. (1990) and Trehub et al. (1986) studies, Trainor and Trehub (1992) suggested that infants younger than 1 year had not acquired sufficient cognitive schemata for musical scales to affect perception. In that study, 8-month-olds and adults were tested for detection of semitone changes in melodies. One type of semitone change was to a note that was within the basic musical key of the melody, and the other type of change was to a note outside of the key. Adults detected the outside-key change better than they detected the withinkey change. Infants detected both changes equivalently well. The adults may have been able to more readily detect the outside-key change because it violated perceptual expectations generated from schematic representations of the functions of notes within musical keys (Krumhansl, 1990). Eight-month-olds may not have possessed schemas for note function, thus allowing them to process the semitone changes independently of musical key knowledge. In the present study, 6-month-olds may have demonstrated knowledge of interval patterns and frequency relations that is acquired before knowledge about how notes function in accordance with musical grammar (Lerdahl \& Jackendoff, 1983). One aspect of this representation could be based on the semitone modulus, because that invariant property of Western music may be among the first to be extracted by infants.

The development of culture-dependent interval processing between 6 months and 1 year requires further investigation. Because the 1-year-olds of Experiment 2 and the 6-month-olds of Experiment 3 were necessarily tested on different degrees of mistuning, it is not clear, for example, whether superior major processing at 1 year is a result of perceptual gain on major scales or a result of perceptual loss on augmented and pelog scales (see Aslin \& Pisoni, 1980 , for review of possible developmental courses of auditory perception). Testing of more narrowly spaced infant age groups on the same amount of mistuning would allow for both comparison of both performance patterns and absolute performance across the age groups. The current 6-month-olds and 1-year-olds were sufficiently far apart in age so that comparison of absolute performance could not be made without confounding of perceptual ability and the 1-year-olds' potentially better management of the task demands of the OHTP. In addition, although the present work has suggested some interesting relations between 1year-olds' and adults' processing of interval structure, there exist substantial gaps in our knowledge of development of musical processing between infancy and adulthood. More emphasis on the study of toddlers, preschoolers, and school-aged children could solve many of the mysteries of the developmental course of music perception, speech perception, and their relations with the ontogeny of other cognitive abilities.

\section{REFERENCES}

Asuin, R. N., Pisoni, D. B. (1980). Some developmental processes in speech perception. In G. H. Yeni-Komshian, J. F. Kavanagh, \& C. A. Ferguson (Eds.), Child phonology: Vol. 2. Perception (pp. 6796). Cambridge: Cambridge University Press.

Balzano, G. J. (1980). The group-theoretic description of 12-fold and microtonal pitch systems. Computer Music Journal, 4(4), 66-84.

BALZANo, G. J. (1982). Musical vs. psychoacoustical variables and their influence on the perception of musical intervals. Bulletin of the Council for Research in Music Education, 70, 1-11.

Balzano, G. J. (1986a). Music perception as detection of pitch-time constraints. In V. McCabe \& G. J. Balzano (Eds.), Event cognition: An ethological perspective (pp. 217-233). Hillsdale, NJ: Erlbaum.

Balzano, G. J. (1986b). What are musical pitch and timbre? Music Perception, 3, 297-314.

Balzano, G. J. (1987). Measuring music. In A. Gabrielsson (Ed.), Action and perception in thythm and music (pp. 177-199). Stockholm: Royal Swedish Academy of Music.

CAMPBE LL, G. (1988). Expansions: A method for developing new material for improvisation. Lebanon, IN: Houston Publishing.

Castellano, M. A., Bharucha, J. J., \& Krumhansl, C. L. (1984). Tonal hierarchies in the music of North India. Journal of Experimental Psychology: General, 113, 394-412.

Cohen, A. J., ThorPe, L. A., \& TrehuB, S. E. (1987). Infants' perception of musical relations in short transposed tone sequences. Canadian Journal of Psychology, 41, 33-47.

Cohen, A. J., Trehub, S. E., \& Thorpe, L. A. (1989). Effects of uncertainty on melodic information processing. Perception \& Psychophysics, 46, 18-28.

Cuddy, L. L., Cohen, A. J., \& Mewhort, D. J. K. (1981). Perception of structure in short melodic sequences. Journal of Experimental Psychology: Human Perception \& Performance, 7, 869-883.

Cuddy, L. L., Cohen, A. J., \& Miller, J. (1979). Melody recognition: The experimental application of musical rules. Canadian Journal of Psychology, 33, 148-157.

Dowling, W. J. (1978). Scale and contour: Two components of a theory of memory for melodies. Psychological Review, 85, 341-354.

Dowling, W. J., \& Harwoon, D. L. (1986). Music cognition. New York: Academic Press.

EILERS, R. E., \& GAVIN, W. J. (1981). The evaluation of infant speech perception skills: Statistical techniques and theory development. In R. E. Stark (Ed.), Language behavior in infancy and early childhood (pp. 185-213). Amsterdam: Elsevier North-Holland.

Eilers, R. E., Wilson, W. R., \& MoORE, J. M. (1977). Developmental changes in speech discrimination in infants. Joumal of Speech \& Hearing Research, 20, 766-780.

Green, D. M., Swets, J. A. (1966). Signal detection theory and psychophysics. New York: Wiley.

Hulse, S. H., \& PAGE, S. C. (1988). Toward a comparative psychology of music perception. Music Perception, 5, 427-452.

Johnson, M. H., Morton, J. (1991). Biology and cognitive development: The case of face recognition. Cambridge, U.K.: Blackwell.

Kessler, E. J., Hansen, C., \& Shepard, R. N. (1984). Tonal schemata in the perception of music in Bali and in the West. Music Perception, 2, 131-165.

KrumhansL, C. L. (1990). Cognitive foundations of musical pitch. Oxford: Oxford University Press.

Krumhansl, C. L., KeIL, F. C. (1982). Acquisition of the hierarchy of tonal functions in music. Memory \& Cognition, 10, 243-251.

Kuhl, P. K., Willams, K. A., Lacerde, F., Stevens, K. N., * LINDBLOM, B. (1992). Linguistic experience alters phonetic perception in infants by 6 months of age. Science, 255, 606-608.

KUNST, J. (1973). Music in Java: Its history, its theory and its technique. The Hague: Martinus Nijhoff.

LERDAHL, F., \& JACKENDOFF, R. (1983). A generative theory of tonal music. Cambridge, MA: MIT Press.

Lynch, M. P., Eilers, R. E., Oller, D. K., Urbano, R. C. (1990). Innateness, experience, and music perception. Psychological Science, 1, 272-276.

Lynch, M. P., Eilers, R. E., Oller, D. K., Urbano, R. C., \& WilSON, P. (1991). Influences of acculturation and musical sophistica- 
tion on perception of musical interval patterns. Journal of Experimental Psychology: Human Perception \& Performance, 17, 967-975.

Macmillan, N. A., \& Kaplan, H. L. (1985). Detection theory analysis of group data: Estimating sensitivity from average hit and falsealarm rates. Psychological Bulletin, 98, 185-199.

MaLM, W. P. (1977). Music cultures of the Pacific, the Near East, and Asia (2nd ed.). Englewood Cliffs, NJ: Prentice-Hall.

Massaro, D. W. (1975). Experimental psychology and information processing. Chicago: Rand McNally.

NetTL, B. (1956). Music in primitive culture. Cambridge, MA: Harvard University Press.

Olsho, L. W., Schoon, C., Sakai, R., Turpin, R., \& Sperduto, V. (1982). Auditory frequency discrimination in infancy. Developmental Psychology, 18, 721-726.

ShePard, R. N., Jordan, D. S. (1984). Auditory illusions demonstrating that tones are assimilated to an internalized musical scale. Science, 226, 1333-1334.

SwEts, J. A. (ED.). (1964). Signal detection and recognition by human observers: Contemporary readings. New York: Wiley.

Thorpe, L. A., Trehub, S. E. (1989). Duration illusion and auditory grouping in infancy. Developmental Psychology, 25, 122-127.

Thorpe, L. A., Trehub, S. E., Morrongiello, B. A., \& Bull, D.
(1988). Perceptual grouping by infants and preschool children. Developmental Psychology, 24, 484-491.

TrainOR, L. J., \& TrehUb, S. E. (1992). A comparison of infants' and adults' sensitivity to Western musical structure. Joumal of Experimental Psychology: Human Perception \& Performance, 18, 394-402.

Trehub, S. E., Cohen, A. J., Thorpe, L. A., Morrongiello, B. A. (1986). Development of the perception of musical relations: Semitone and diatonic structure. Journal of Experimental Psychology: Human Perception \& Performance, 12, 295-301.

Trehub, S. E., Thorpe, L. A., \& Trainor, L. J. (1990). Infants' perception of good and bad melodies. Psychomusicology, 9, 5-15.

Werker, J. F., Gilbert, J. H. V., Humphrey, K., \& Tees, R. C. (1981). Developmental aspects of cross-language speech perception. Child Development, 52, 349-355.

WERKER, J. F., \& TEEs, R. C. (1984). Cross-language speech perception: Evidence for perceptual reorganization during the first year of life. Infant Behavior \& Development, 7, 49-63.

(Manuscript received December 11, 1991; revision accepted for publication June 6, 1992.) 